\title{
INTERIOR CAUCHY-SCHAUDER ESTIMATES FOR THE HEAT FLOW IN CARNOT-CARATHÉODORY SPACES*
}

\author{
DONATELLA DANIELLI ${ }^{\dagger}$ AND NICOLA GAROFALO ${ }^{\ddagger}$ \\ Dedicated to Neil Trudinger, with friendship and deep admiration, \\ on the occasion of his 65th birthday
}

Key words. Carnot-Carathéodory spaces, Heat equation, Interior Schauder estimates.

AMS subject classifications. 35K65, 35B45

1. Introduction. The purpose of this paper is to establish some basic interior estimates of Cauchy-Schauder type for the heat flow associated with a system $X=$ $\left\{X_{1}, \ldots, X_{m}\right\}$ of $C^{\infty}$ vector fields in $\mathbb{R}^{n}$ satisfying Hörmander's finite rank condition $[\mathrm{H}]$

$$
\operatorname{rank} \operatorname{Lie}\left[X_{1}, \ldots, X_{m}\right] \equiv n .
$$

Besides from playing an important role in the applications, such estimates also have an obvious independent interest. Similarly to the classical ones for elliptic equations, our results are tailored on the intrinsic geometry of the system $X=\left\{X_{1}, \ldots, X_{m}\right\}$. We consider the sub-Laplacian associated with $X$

$$
\mathcal{L}=-\sum_{j=1}^{m} X_{j}^{*} X_{j},
$$

and the corresponding heat operator in $\mathbb{R}^{n+1}$

$$
\mathcal{H}=\mathcal{L}-\frac{\partial}{\partial t} .
$$

Thanks to Hörmander's theorem [H], the assumption (1.1) guarantees the hypoellipticity of both $\mathcal{L}$ and $\mathcal{H}$ on their respective domains. Therefore, distributional solutions of either $\mathcal{L} u=f$, or $\mathcal{H} u=F$ are $C^{\infty}$, wherever such are $f$ or $F$. Using the basic results in [RS], the existence of a fundamental solution for $\mathcal{L}$ and its size estimates were established independently by Sanchez-Calle [SC] and by Nagel, Stein and Wainger $[\mathrm{NSW}$. Corresponding Gaussian estimates for the heat kernels were independently obtained by Jerison and Sanchez-Calle [JSC] and by Kusuoka and Stroock [KS1], [KS2]. One should also see [VSC].

To state our main result we define for $z=(x, t) \in \mathbb{R}^{n+1}$ and $r>0$ the parabolic cylinders

$$
Q(z, r)=B(x, r) \times\left(t-r^{2}, t\right), \quad Q^{-}(z, r)=B(x, r) \times\left(t-r^{2}, t-\frac{r^{2}}{4}\right),
$$

\footnotetext{
* Received May 14, 2008; accepted for publication June 13, 2008.

${ }^{\dagger}$ Department of Mathematics, Purdue University, West Lafayette, IN 47907, USA (danielli@ math.purdue.edu). First author supported in part by NSF CAREER Grant, DMS-0239771.

${ }_{\ddagger}^{\ddagger}$ Department of Mathematics, Purdue University, West Lafayette, IN 47907, USA (garofalo@ math.purdue.edu); Dipartimento di Metodi e Modelli Matematici per le Scienze Applicate, Università di Padova, 35131 Padova, Italy (garofalo@dmsa.unipd.it). Second author supported in part by NSF Grant DMS-0701001.
} 
where we have indicated by $B(x, r)=\left\{y \in \mathbb{R}^{n} \mid d(x, y)<r\right\}$ the metric ball in the Carnot-Carathéodory $(\mathrm{CC})$ distance associated with $X$. Slightly abusing the notation we will write $|B(x, r)|$ for the $n$-dimensional Lebesgue measure of the ball $B(x, r)$ in $\mathbb{R}^{n}$, and also $|Q(z, r)|$ for the $(n+1)$-dimensional Lebesgue measure of the cylinder $Q(z, r)$ in $\mathbb{R}^{n+1}$. We note explicitly that $|Q(z, r)|=r^{2}|B(x, r)|$. Here is the main result in this paper.

TheOrem 1.1. Let $D \subset \mathbb{R}^{n+1}$ be an open set and suppose that $u$ solves $\mathcal{H} u=0$ in $D$. There exists $R_{o}>0$, depending on $D$ and $X$, such that for every $z_{o} \in D$ and $0<r \leq R_{o}$ for which $\bar{Q}\left(z_{o}, r\right) \subset D$, one has for any $s, k \in \mathbb{N} \cup\{0\}$

$$
\sup _{Q\left(z_{o}, r / 2\right)}\left|\frac{\partial^{k}}{\partial t^{k}} X_{j_{1}} X_{j_{2}} \ldots X_{j_{s}} u\right| \leq \frac{C}{r^{s+2 k}} \frac{1}{\left|Q\left(z_{o}, 2 r\right)\right|} \int_{Q\left(z_{o}, 2 r\right)}|u| d \xi d \tau
$$

for some constant $C=C(D, X, s, k)>0$. In the above estimate, for every $i=1, \ldots, s$, the index $j_{i}$ runs in the set $\{1, \ldots, m\}$. When $u \geq 0$ in $D$, then the above estimate can be replaced by

$$
\sup _{Q^{-}\left(z_{o}, r / 2\right)}\left|\frac{\partial^{k}}{\partial t^{k}} X_{j_{1}} X_{j_{2}} \ldots X_{j_{s}} u\right| \leq \frac{C}{r^{s+2 k}} u\left(z_{o}\right) .
$$

Theorem 1.1 generalizes (and contains) the following steady-state CauchySchauder type estimates which were found in [CGN3].

TheOrem 1.2. Let $\Omega \subset \mathbb{R}^{n}$ be a bounded open set and suppose that $\mathcal{L} u=0$ in $\Omega$. There exists $R_{o}>0$, depending on $\Omega$ and $X$, such that for every $x \in \Omega$ and $0<r \leq R_{o}$ for which $\bar{B}(x, r) \subset \Omega$, one has for any $s \in \mathbb{N}$

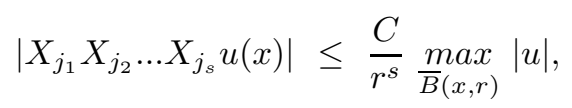

for some constant $C=C(\Omega, X, s)>0$. In the above estimate, for every $i=1, \ldots, s$, the index $j_{i}$ runs in the set $\{1, \ldots, m\}$.

This result was proved in [CGN3] with a different approach which exploited the geometry of the level sets of the fundamental solution of the relevant sub-Laplacian. That approach however does not seem to work in the time-dependent setting of this paper since one presently lacks some very delicate asymptotic estimates of the relevant heat kernels. In the special setting of the Heisenberg group $\mathbb{H}^{n}$ such estimates were obtained in [GS] using a refined asymptotic analysis of the Fourier integrals involved in Gaveau's explicit fundamental solution for the heat equation, see [Ga]. But the explicit calculations in [GS] are out of question in the general setting of this paper. We have been able to get around such lack of estimates by: (i) working with intrinsic parabolic cylinders in which the base is not a Carnot-Carathéodory ball, but rather a level set of the steady-state fundamental solution. This allows us to construct some appropriate $C^{\infty}$ cut-off functions, see Lemma 3.2; (ii) mimicking the basic idea in E. E. Levi's method of the parametrix.

To see that Theorem 1.1 contains Theorem 1.2 it suffices to apply the former result to the function $u(x, t)=u(x)$, where $u(x)$ solves $\mathcal{L} u=0$ in $\Omega \subset \mathbb{R}^{n}$. It is worth emphasizing that, in contrast with the classical case, in the subelliptic setting any derivative $X_{j_{i}} u$ of a solution to $\mathcal{H} u=0$ fails to be itself a solution of $\mathcal{H} u=0$. Our 
proof of Theorem 1.1 is based on the Gaussian estimates of the heat kernel found in [JSC], [KS1], [KS2]. In particular, we will assume such estimates in the version which was obtained in [KS1]. With such basic tool in hands, we will then use arguments which are reminiscent of the classical ones originated with E. E. Levi's method of the parametrix, see $[\mathrm{Fr}]$, or also $[\mathrm{E}]$. It is worth mentioning at this point that, in the special setting of Carnot groups, our arguments combined with the non-isotropic group dilations, provide a simplified approach to several of the local and global results available in the literature.

Similarly to its classical ancestor, Theorem 1.1 has many basic consequences. For instance, one can use it to study the regularity at the boundary for solutions to the equation $\mathcal{H} u=0$ both in cylindrical and non-cylindrical domains in $\mathbb{R}^{n+1}$. Basic applications of the Cauchy-Schauder estimates in the time-independent subelliptic Dirichlet problem can be found in the works [CGN1], [LU1], [CGN2], [LU2], [CGN3]. In a different direction, such estimates were used in the works [CDG1], [CDG2], [CDG3]. Theorem 1.1 can also be used to deduce some interesting Cauchy-Liouville type properties of solutions to parabolic equations such as (1.3).

2. Preliminaries. In $\mathbb{R}^{n}$, with $n \geq 3$, we consider a system $X=\left\{X_{1}, \ldots, X_{m}\right\}$ of $C^{\infty}$ vector fields satisfying Hörmander finite rank condition. A piecewise $C^{1}$ curve $\gamma:[0, T] \rightarrow \mathbb{R}^{n}$ is called sub-unitary if whenever $\gamma^{\prime}(t)$ exists one has for every $\xi \in \mathbb{R}^{n}$

$$
<\gamma^{\prime}(t), \xi>^{2} \leq \sum_{j=1}^{m}<X_{j}(\gamma(t)), \xi>^{2} .
$$

We note explicitly that the above inequality forces $\gamma^{\prime}(t)$ to belong to the span of $\left\{X_{1}(\gamma(t)), \ldots, X_{m}(\gamma(t))\right\}$. The sub-unit length of $\gamma$ is by definition $l_{s}(\gamma)=T$. Given $x, y \in \mathbb{R}^{n}$, denote by $\mathcal{S}_{\Omega}(x, y)$ the collection of all sub-unitary $\gamma:[0, T] \rightarrow \Omega$ which join $x$ to $y$. The Chow-Rashevsky accessibility theorem states that, given a connected open set $\Omega \subset \mathbb{R}^{n}$, for every $x, y \in \Omega$ there exists $\gamma \in \mathcal{S}_{\Omega}(x, y)$, see [Ch], [Ra]. As a consequence, if we pose

$$
d_{\Omega}(x, y)=\inf \left\{l_{s}(\gamma) \mid \gamma \in \mathcal{S}_{\Omega}(x, y)\right\},
$$

we obtain a distance on $\Omega$, called the Carnot-Carathéodory distance on $\Omega$, associated with the system $X$. When $\Omega=\mathbb{R}^{n}$, we write $d(x, y)$ instead of $d_{\mathbb{R}^{n}}(x, y)$. It is clear that $d(x, y) \leq d_{\Omega}(x, y), x, y \in \Omega$, for every connected open set $\Omega \subset \mathbb{R}^{n}$. In [NSW] it was proved that for every connected $\Omega \subset \subset \mathbb{R}^{n}$ there exist $C, \epsilon>0$ such that

$$
C|x-y| \leq d_{\Omega}(x, y) \leq C^{-1}|x-y|^{\epsilon}, \quad x, y \in \Omega,
$$

see also [Be]. This gives $d(x, y) \leq C^{-1}|x-y|^{\epsilon}, x, y \in \Omega$, and therefore

$$
i:\left(\mathbb{R}^{n},|\cdot|\right) \rightarrow\left(\mathbb{R}^{n}, d\right) \quad \text { is continuous. }
$$

It is easy to see that also the continuity of the opposite inclusion holds [GN1], hence the metric and the Euclidean topology are compatible.

For $x \in \mathbb{R}^{n}$ and $r>0$, we let $B_{d}(x, r)=\left\{y \in \mathbb{R}^{n} \mid d(x, y)<r\right\}$. The basic properties of these balls were established by Nagel, Stein and Wainger in their seminal paper [NSW]. Denote by $Y_{1}, \ldots, Y_{l}$ the collection of the $X_{j}$ 's and of those commutators which are needed to generate $\mathbb{R}^{n}$. A formal "degree" is assigned to each $Y_{i}$, namely the corresponding order of the commutator. If $I=\left(i_{1}, \ldots, i_{n}\right), 1 \leq i_{j} \leq l$ is a $n$-tuple of 
integers, following [NSW] we let $d(I)=\sum_{j=1}^{n} \operatorname{deg}\left(Y_{i_{j}}\right)$, and $a_{I}(x)=\operatorname{det}\left(Y_{i_{1}}, \ldots, Y_{i_{n}}\right)$. The Nagel-Stein-Wainger polynomial is defined by

$$
\Lambda(x, r)=\sum_{I}\left|a_{I}(x)\right| r^{d(I)}, \quad r>0 .
$$

For a given bounded open set $U \subset \mathbb{R}^{n}$, we let

$$
Q=\sup \left\{d(I)|| a_{I}(x) \mid \neq 0, x \in U\right\}, \quad Q(x)=\inf \left\{d(I)|| a_{I}(x) \mid \neq 0\right\},
$$

and notice that $n \leq Q(x) \leq Q$. The numbers $Q$ and $Q(x)$ are respectively called the local homogeneous dimension of $U$ and the homogeneous dimension at $x$ with respect to the system $X$.

TheOREM 2.1 ([NSW]). For every bounded set $U \subset \mathbb{R}^{n}$, there exist constants $C, R_{o}>0$ such that, for any $x \in U$, and $0<r \leq R_{o}$,

$$
C \Lambda(x, r) \leq\left|B_{d}(x, r)\right| \leq C^{-1} \Lambda(x, r) .
$$

As a consequence, one has with $C_{1}=2^{Q}$

$$
\left|B_{d}(x, 2 r)\right| \leq C_{1}\left|B_{d}(x, r)\right| \quad \text { for every } \quad x \in U \text { and } 0<r \leq R_{o} .
$$

The numbers $C_{1}, R_{o}$ in (2.5) will be referred to as the characteristic local parameters of $U$. We notice that (2.5) implies for every $x \in U, 0<r \leq R_{o}$ and $0 \leq t \leq 1$

$$
|B(x, t r)| \geq C_{1} t^{Q}|B(x, r)| .
$$

Because of (2.2), if we let

$$
E(x, r)=\frac{\Lambda(x, r)}{r^{2}}
$$

then the function $r \rightarrow E(x, r)$ is strictly increasing. We denote by $F(x, \cdot)$ the inverse function of $E(x, \cdot)$, so that $F(x, E(x, r))=r$. Let $\Gamma(x, y)=\Gamma(y, x)$ be the positive fundamental solution of the sub-Laplacian

$$
\mathcal{L}=\sum_{j=1}^{m} X_{j}^{*} X_{j},
$$

and consider its level sets

$$
\Omega(x, r)=\left\{y \in \mathbb{R}^{n} \mid \Gamma(x, y)>\frac{1}{r}\right\} .
$$

The following definition plays a key role in this paper.

Definition 2.2. For every $x \in \mathbb{R}^{n}$, and $r>0$, the set

$$
B_{X}(x, r)=\left\{y \in \mathbb{R}^{n} \mid \Gamma(x, y)>\frac{1}{E(x, r)}\right\}
$$

will be called the $X$-ball, centered at $x$ with radius $r$. 
We note explicitly that

$$
B_{X}(x, r)=\Omega(x, E(x, r)), \quad \text { and that } \quad \Omega(x, r)=B_{X}(x, F(x, r)) .
$$

One of the main geometric properties of the $X$-balls, is that they are equivalent to the Carnot-Carathéodory balls. To see this, we recall the following important result, established independently in $[\mathrm{NSW}],[\mathrm{SC}]$, see also $[\mathrm{FSC}]$. Hereafter, the notation $X u=\left(X_{1} u, \ldots, X_{m} u\right)$ indicates the sub-gradient of a function $u$, whereas $|X u|=$ $\left(\sum_{j=1}^{m}\left(X_{j} u\right)^{2}\right)^{\frac{1}{2}}$ will denote its length.

TheOREM 2.3. Given a bounded set $U \subset \mathbb{R}^{n}$, there exists $R_{o}$, depending on $U$ and on $X$, such that for $x \in U, 0<d(x, y) \leq R_{o}$, one has for $s \in \mathbb{N} \cup\{0\}$, and for some constant $C=C(U, X, s)>0$

$$
\begin{aligned}
& \left|X_{j_{1}} X_{j_{2}} \ldots X_{j_{s}} \Gamma(x, y)\right| \leq C^{-1} \frac{d(x, y)^{2-s}}{\left|B_{d}(x, d(x, y))\right|}, \\
& \Gamma(x, y) \geq C \frac{d(x, y)^{2}}{\left|B_{d}(x, d(x, y))\right|} .
\end{aligned}
$$

In the first inequality in (2.8), one has $j_{i} \in\{1, \ldots, m\}$ for $i=1, \ldots, s$, and $X_{j_{i}}$ is allowed to act on either $x$ or $y$.

In view of $(2.5),(2.8)$, it is now easy to recognize that, given a bounded set $U \subset \mathbb{R}^{n}$, there exists $a>1$, depending on $U$ and $X$, such that

$$
B\left(x, a^{-1} r\right) \subset B_{X}(x, r) \subset B(x, a r),
$$

for $x \in U, 0<r \leq R_{o}$. We observe that, as a consequence of (2.4), and of (2.8), one has

$$
C d(x, y) \leq F\left(x, \frac{1}{\Gamma(x, y)}\right) \leq C^{-1} d(x, y),
$$

for all $x \in U, 0<d(x, y) \leq R_{o}$.

We observe that for a Carnot group $\boldsymbol{G}$ of step $k$, if $\mathfrak{g}=V_{1} \oplus \ldots \oplus V_{k}$ is a stratification of the Lie algebra of $\boldsymbol{G}$, then one has $\Lambda(x, r)=$ const $r^{Q}$, for every $x \in \boldsymbol{G}$ and every $r>0$, with $Q=\sum_{j=1}^{k} j \operatorname{dim} V_{j}$, the homogeneous dimension of the group $\boldsymbol{G}$. In this case $Q(x) \equiv Q$, see [F], [BLU], [G].

It is important to keep in mind the following basic properties of a CarnotCarathéodory space.

Proposition 2.4. $\left(\mathbb{R}^{n}, d\right)$ is locally compact. Furthermore, for any bounded set $U \subset \mathbb{R}^{n}$ there exists $R_{o}=R_{o}(U)>0$ such that the closed balls $\bar{B}\left(x_{o}, R\right)$, with $x_{o} \in U$ and $0<R<R_{o}$, are compact.

REMARK 2.5. Compactness of balls of large radii may fail in general, see [GN1]. However, there are important cases in which Proposition 2.4 holds globally, in the sense that one can take $U$ to coincide with the whole ambient space and $R_{o}=\infty$. One example is that of Carnot groups. Another interesting case is that when the vector fields $X_{j}$ have coefficients which are globally Lipschitz, see [GN1], [GN2]. Henceforth, 
for any given bounded set $U \subset \mathbb{R}^{n}$ we will always assume that the local parameter $R_{o}$ has been chosen so to accommodate Proposition 2.4.

Keeping in mind the applicability of the results in this paper to the study of the Dirichlet problem, the basic reference on this subject for operators of Hörmander type remains the pioneer study of Bony $[\mathrm{B}]$. For more recent developments the reader should consult [CG], [CGN3] and the references therein.

The following basic result was established in [KS1], [KS2], see also [JSC].

THEOREM 2.6. The fundamental solution $p(x, t ; \xi, \tau)=p(x, \xi ; t-\tau)$ with singularity at $(\xi, \tau)$ satisfies the following size estimates: there exists $M=M(X)>0$ and for every $k, s \in \mathbb{N} \cup\{0\}$, there exists a constant $C=C(X, k, s)>0$, such that

$$
\begin{aligned}
& \left|\frac{\partial^{k}}{\partial t^{k}} X_{j_{1}} X_{j_{2}} \ldots X_{j_{s}} p(x, t ; \xi, \tau)\right| \leq \frac{C}{(t-\tau)^{s+2 k}} \frac{1}{|B(x, \sqrt{t-\tau})|} \exp \left(-\frac{M d(x, \xi)^{2}}{t-\tau}\right), \\
& (2.12) \quad p(x, t ; \xi, \tau) \geq \frac{C^{-1}}{|B(x, \sqrt{t-\tau})|} \exp \left(-\frac{M^{-1} d(x, \xi)^{2}}{t-\tau}\right),
\end{aligned}
$$

for every $x, \xi \in \mathbb{R}^{n}$, and any $-\infty<\tau<t<\infty$.

3. Proof of Theorem 1.1. In the sequel we fix a point $z_{o}=\left(x_{o}, t_{o}\right) \in \mathbb{R}^{n+1}$ and consider the following parabolic cylinders

$$
\begin{gathered}
Q\left(z_{o}, r\right)=B\left(x_{o}, r\right) \times\left(t_{o}-r^{2}, t_{o}\right), \\
Q^{-}\left(z_{o}, r\right)=B\left(x_{o}, r\right) \times\left(t_{o}-r^{2}, t_{o}-\frac{r^{2}}{4}\right) .
\end{gathered}
$$

We will also need cylinders based on the $X$-balls

$$
\begin{gathered}
Q_{X}\left(z_{o}, r\right)=B_{X}\left(x_{o}, r\right) \times\left(t_{o}-r^{2}, t_{o}\right), \\
Q_{X}^{-}\left(z_{o}, r\right)=B_{X}\left(x_{o}, r\right) \times\left(t_{o}-r^{2}, t_{o}-\frac{r^{2}}{4}\right) .
\end{gathered}
$$

The following basic lemma, which constitutes a generalization of a result obtained in [CGL] for the case $s=1$, is the main motivation for introducing the cylinders $Q_{X}\left(z_{o}, r\right)$. If we work with the $C C$ balls $B\left(x_{o}, r\right)$ then the existence of a smooth cut-off function fails since in general only the first derivatives of the $C C$ distance with respect to the vector fields $X_{1}, \ldots, X_{m}$ are bounded, see e.g. [GN2].

Lemma 3.1. For every $x_{o} \in \mathbb{R}^{n}$ and $r>0$ there exists a function $\chi \in$ $C_{o}^{\infty}\left(B_{X}\left(x_{o}, 2 r\right)\right), 0 \leq \chi \leq 1$, such that $\chi \equiv 1$ on $B_{X}\left(x_{o}, r\right)$, and moreover for each $s \in \mathbb{N} \cup\{0\}$ there exists $C=C(X, s)>0$ such that

$$
\left|X_{j_{1}} X_{j_{2}} \ldots X_{j_{s}} \chi\right| \leq \frac{C}{r^{s}} .
$$


Proof. Let $f \in C_{o}^{\infty}([0, \infty)), 0 \leq f \leq 1, f \equiv 1$ on $[0, r]$, supp $f \subset[0,2 r)$, and such that for every $s \in \mathbb{N}$ one has

$$
\left|f^{(s)}(\sigma)\right| \leq \frac{C(s)}{r^{s}} \quad \text { for every } \quad \sigma \in[r, 2 r] .
$$

We define $\chi(x)=f\left(\rho_{x_{o}}(x)\right)$. This function clearly possesses the desired support properties. The estimates for the derivatives of $\chi$ along the vector fields $X_{1}, \ldots, X_{m}$ now follow by recurrence from (3.5) and from the following estimates for the regularized distance $\rho_{x_{o}}$

$$
\left|X_{j_{1}} X_{j_{2}} \ldots X_{j_{s}} \rho_{x_{o}}(x)\right| \leq \frac{C(s)}{\rho_{x_{o}}(x)^{s-1}} \quad x \neq x_{o} .
$$

We leave the details to the reader.

Using Lemma 3.1 we now obtain a similar ad hoc result on the parabolic cylinders $Q_{X}\left(z_{o}, r\right)$.

Lemma 3.2. For every $z_{o}=\left(x_{o}, t_{o}\right) \in \mathbb{R}^{n+1}$ and $r>0$ there exists a function

$$
\zeta \in C_{o}^{\infty}\left(B_{X}\left(x_{o}, 2 r\right) \times\left(t_{o}-4 r^{2}, t_{o}\right]\right)
$$

$0 \leq \zeta \leq 1$, such that $\zeta \equiv 1$ on $\bar{Q}_{X}\left(z_{o}, r\right)$, and moreover for each $s, k \in \mathbb{N} \cup\{0\}$ there exists $C=C(X, s, k)>0$ such that

$$
\left|\frac{\partial^{k}}{\partial t^{k}} X_{j_{1}} X_{j_{2}} \ldots X_{j_{s}} \zeta\right| \leq \frac{C}{r^{s+2 k}} .
$$

Proof. We choose a function $h \in C^{\infty}(\mathbb{R})$, such that $0 \leq h \leq 1, h \equiv 1$ on $\left[t_{o}-r^{2}, \infty\right)$, supp $h \subset\left(t_{o}-4 r^{2}, \infty\right)$, and for which one has for every $k \in \mathbb{N}$

$$
\left|h^{(k)}(t)\right| \leq \frac{C(k)}{r^{2 k}} \quad \text { for every } \quad t \in\left[t_{o}-4 r^{2}, t_{o}-r^{2}\right]
$$

We define

$$
\zeta(z)=\zeta(x, t)=\chi(x) h(t)
$$

where $\chi$ is the function in Lemma 3.1. From the definitions of $\chi$ and $h$ it is clear that $\zeta \equiv 1$ on $\bar{Q}_{X}\left(z_{o}, r\right)$. Since

$$
\left|\frac{\partial^{k}}{\partial t^{k}} X_{j_{1}} X_{j_{2}} \ldots X_{j_{s}} \zeta(z)\right|=\left|X_{j_{1}} X_{j_{2}} \ldots X_{j_{s}} \chi(x)\right|\left|h^{(k)}(t)\right|,
$$

the estimate (3.7) follows from the corresponding ones for $\chi$ and $h$.

We will need the following form of Duhamel's principle.

Proposition 3.3. Let $F \in C_{o}^{\infty}\left(\mathbb{R}^{n} \times[0, \infty)\right)$, and define

$$
w(z)=\int_{0}^{t} \int_{\mathbb{R}^{n}} p(x, \xi ; t-\tau) F(\xi, \tau) d \xi d \tau,
$$


where $p(x, \xi ; t-\tau)$ denotes the positive fundamental solution of $\mathcal{H}$ with singularity at $(\xi, \tau)$. One has $w \in C^{\infty}\left(\mathbb{R}^{n} \times(0, \infty)\right)$, and moreover

$$
\mathcal{H} w=-F \quad \text { in } \mathbb{R}^{n} \times\left(0, t_{o}\right), \quad w(x, 0)=0, \quad x \in \mathbb{R}^{n} .
$$

Proof. Follows along classical lines.

In what follows, given an open set $D \subset \mathbb{R}^{n+1}$, we indicate with $\Gamma^{2,1}(D)$ the collection of all continuous functions on $D$ possessing two continuous derivatives with respect to the vector fields $X_{1}, \ldots, X_{m}$, and one continuous derivative with respect to the variable $t$.

TheOrem 3.4. There exists at most one solution $u \in \Gamma^{2,1}\left(\mathbb{R}^{n} \times\left(0, T_{1}\right]\right)$ to the differential inequality

$$
u(x, t) \mathcal{H} u(x, t) \geq 0 \quad(x, t) \in \mathbb{R}^{n} \times\left(0, T_{1}\right),
$$

such that $u(x, 0)=0$ for $x \in \mathbb{R}^{n}$, and for which

$$
|u(x, t)| p\left(x_{o}, x ; 1\right) \leq A, \quad x \in \mathbb{R}^{n}, 0<t<T_{1},
$$

for some $A>0$, and some $x_{o} \in \mathbb{R}^{n}$. In particular, given $\phi \in C\left(\mathbb{R}^{n}\right), F \in C\left(\mathbb{R}^{n} \times\right.$ $\left.\left(0, T_{1}\right)\right)$, there exists a unique solution to the Cauchy problem

$$
\mathcal{H} u=F \quad \text { in } \mathbb{R}^{n} \times\left(0, T_{1}\right), \quad u(x, 0)=\phi(x), \quad x \in \mathbb{R}^{n},
$$

satisfying the constraint (3.11).

Proof. We introduce the function

$$
\phi(R) \stackrel{\text { def }}{=} \int_{\mathbb{R}^{n}} u^{2}\left(\xi, R^{2}\right) p\left(x, \xi ; T-R^{2}\right) d \xi, \quad 0 \leq R<\sqrt{T},
$$

where $x \in \mathbb{R}^{n}$ is fixed and the number $T<T_{1}$ will be chosen suitably small in a moment. We have from (3.11) and the Gaussian estimates (2.11), (2.12) in Theorem 2.6

$$
\begin{aligned}
& u^{2}\left(\xi, R^{2}\right) p\left(x, \xi ; T-R^{2}\right) \leq A^{2} p\left(x_{o}, \xi ; 1\right)^{-2} p\left(x, \xi ; T-R^{2}\right) \\
& \leq C^{3} A^{2} \frac{\left|B\left(x_{o}, 1\right)\right|^{2}}{\left|B\left(x, \sqrt{T-R^{2}}\right)\right|} \exp \left\{\frac{2 d\left(x_{o}, \xi\right)^{2}}{M}\right\} \exp \left\{-\frac{M d(x, \xi)^{2}}{T-R^{2}}\right\} .
\end{aligned}
$$

At this point we choose

$$
T_{o}<\min \left\{T_{1}, \frac{M^{2}}{4}\right\}
$$

and let $0<T<T_{o}$. Since the triangle inequality gives

$$
d\left(x_{o}, \xi\right)^{2} \leq d\left(x_{o}, x\right)^{2}+d(x, \xi)^{2}+2 d\left(x_{o}, x\right) d(x, \xi),
$$


we obtain from (3.14)

$$
\begin{aligned}
& u^{2}\left(\xi, R^{2}\right) p\left(x, \xi ; T-R^{2}\right) \leq C^{3} A^{2} \frac{\left|B\left(x_{o}, 1\right)\right|^{2}}{\left|B\left(x, \sqrt{T-R^{2}}\right)\right|} \exp \left\{\frac{2 d\left(x_{o}, x\right)^{2}}{M}\right\} \\
& \times \exp \left\{\frac{2 d(x, \xi)^{2}}{M}+\frac{4 d\left(x_{o}, x\right) d(x, \xi)}{M}\right\} \exp \left\{-\frac{M d(x, \xi)^{2}}{T}\right\} . \\
& \leq C^{3} A^{2} \frac{\left|B\left(x_{o}, 1\right)\right|^{2}}{\left|B\left(x, \sqrt{T-R^{2}}\right)\right|} \exp \left\{\frac{2 d\left(x_{o}, x\right)^{2}}{M}\right\} \\
& \times \exp \left\{\frac{2 d(x, \xi)^{2}}{M}+\frac{4 d\left(x_{o}, x\right) d(x, \xi)}{M}\right\} \exp \left\{-\frac{4 d(x, \xi)^{2}}{M}\right\},
\end{aligned}
$$

where in the last inequality we have used (3.15). From (3.16) it is clear that if $d(x, \xi) \geq 4 d\left(x_{o}, x\right)$, then

$$
\begin{aligned}
& u^{2}\left(\xi, R^{2}\right) p\left(x, \xi ; T-R^{2}\right) \\
& \leq C^{3} A^{2} \frac{\left|B\left(x_{o}, 1\right)\right|^{2}}{\left|B\left(x, \sqrt{T-R^{2}}\right)\right|} \exp \left\{\frac{2 d\left(x_{o}, x\right)^{2}}{M}\right\} \exp \left\{-\frac{d(x, \xi)^{2}}{M}\right\} .
\end{aligned}
$$

From this estimate we infer that the integral in (3.13) defining $\phi(R)$ is absolutely convergent for any $x \in \mathbb{R}^{n}$ and every $0<T<T_{o}$, with $T_{o}$ satisfying (3.15).

Differentiating under the integral sign we find

$$
\begin{aligned}
\phi^{\prime}(R) & =4 R \int_{\mathbb{R}^{n}} u\left(\xi, R^{2}\right) \partial_{t} u\left(\xi, R^{2}\right) p\left(x, \xi ; T-R^{2}\right) d \xi \\
& -2 R \int_{\mathbb{R}^{n}} u^{2}\left(\xi, R^{2}\right) \partial_{t} p\left(x, \xi ; T-R^{2}\right) d \xi .
\end{aligned}
$$

We now use the self-adjointness of the sub-Laplacian $\mathcal{L}$, and the equation satisfied by $p$, which gives for every $x, \xi \in \mathbb{R}^{n}$ and every $R \in(0, \sqrt{T})$

$$
\partial_{t} p\left(x, \xi ; T-R^{2}\right)=\mathcal{L}_{\xi} p\left(x, \xi ; T-R^{2}\right) .
$$

Substituting the latter equation in the right-hand side of (3.17) we obtain

$$
\begin{aligned}
\phi^{\prime}(R) & =4 R \int_{\mathbb{R}^{n}} u\left(\xi, R^{2}\right) \partial_{t} u\left(\xi, R^{2}\right) p\left(x, \xi ; T-R^{2}\right) d \xi \\
& -2 R \int_{\mathbb{R}^{n}} u^{2}\left(\xi, R^{2}\right) \mathcal{L}_{\xi} p\left(x, \xi ; T-R^{2}\right) d \xi .
\end{aligned}
$$

We now integrate by parts in the second integral in the right-hand side of (3.18). Using the assumption (3.11) we conclude that

$$
\begin{aligned}
\phi^{\prime}(R) & =4 R \int_{\mathbb{R}^{n}} u\left(\xi, R^{2}\right) \partial_{t} u\left(\xi, R^{2}\right) p\left(x, \xi ; T-R^{2}\right) d \xi \\
& -2 R \int_{\mathbb{R}^{n}} \mathcal{L}_{\xi}\left(u^{2}\left(\xi, R^{2}\right)\right) p\left(x, \xi ; T-R^{2}\right) d \xi .
\end{aligned}
$$

Observe that

$$
\mathcal{L}_{\xi}\left(u^{2}\left(\cdot, R^{2}\right)\right)(\xi)=2 u\left(\xi, R^{2}\right) \mathcal{L} u\left(\xi, R^{2}\right)+2\left|X u\left(\xi, R^{2}\right)\right|^{2} .
$$


Substitution in the latter equation gives

$$
\begin{aligned}
\phi^{\prime}(R) & =-4 R \int_{\mathbb{R}^{n}} u\left(\xi, R^{2}\right) \mathcal{H} u\left(\xi, R^{2}\right) p\left(x, \xi ; T-R^{2}\right) d \xi \\
& -4 R \int_{\mathbb{R}^{n}}\left|X u\left(\xi, R^{2}\right)\right|^{2} p\left(x, \xi ; T-R^{2}\right) d \xi .
\end{aligned}
$$

If we now use the hypothesis (3.10), then we conclude from (3.20)

$$
\phi^{\prime}(R) \leq-4 R \int_{\mathbb{R}^{n}}\left|X u\left(\xi, R^{2}\right)\right|^{2} p\left(x, \xi ; T-R^{2}\right) d \xi \leq 0,
$$

and therefore the function $\phi(R)$ is decreasing. In particular, for every $0<R<\sqrt{T}$ one has

$$
\phi(R) \leq \phi(0)=\int_{\mathbb{R}^{n}} u(\xi, 0)^{2} p(x, \xi ; T) d \xi=0 .
$$
we infer

Letting $R \rightarrow \sqrt{T}$ in the latter inequality, by the $\delta$-function property of $p(x, \xi ; \cdot)$

$$
\lim _{R \rightarrow \sqrt{T}} \phi(R)=u(x, T)^{2}=0 .
$$

By the arbitrariness of $x \in \mathbb{R}^{n}, T \in\left(0, T_{o}\right)$, we conclude that it must be $u \equiv 0$ in $\mathbb{R}^{n} \times\left(0, T_{o}\right)$. Repeating the above arguments on successive intervals $\left(T_{o}, 2 T_{o}\right)$, etc., we finally reach the conclusion $u \equiv 0$ in $\mathbb{R}^{n} \times\left(0, T_{1}\right)$.

To prove the second part of the theorem, we observe that by linearity it suffices to show that if $\mathcal{H} u=0$ in $\mathbb{R}^{n} \times\left(0, T_{1}\right), u(x, 0) \equiv 0$, and $u$ satisfies the constraint (3.11), then it must be $u \equiv 0$ in $\mathbb{R}^{n} \times\left(0, T_{1}\right)$. This is of course an immediate consequence of the first part.

We are now ready to establish the main result of this section.

Proof of Theorem 1.1. Without loss of generality we assume that $t_{o}>0$, and consider $r>0$ such that $t_{o}-4 r^{2}>0$. For a given function $u \in C^{\infty}\left(\mathbb{R}^{n+1}\right)$ we define

$$
v(z) \stackrel{\text { def }}{=} \zeta(z) u(z)
$$

where $\zeta$ is the cut-off whose existence has been established in Lemma 3.2. One has

$$
\mathcal{H} v=u \mathcal{H} \zeta+\zeta \mathcal{H} u+2<X u, X \zeta>,
$$

and also, thanks to the support properties of $\zeta$, we have

$$
v(x, 0)=0 \quad x \in \mathbb{R}^{n} .
$$

Suppose that $u$ be a solution to $\mathcal{H} u=0$, then we obtain from (3.23)

$$
\mathcal{H} v=u \mathcal{H} \zeta+2<X u, X \zeta>\stackrel{\text { def }}{=}-F \quad \text { in } \quad \mathbb{R}^{n} \times\left(0, t_{o}\right) .
$$

We next define

$$
w(z)=\int_{0}^{t} \int_{\mathbb{R}^{n}} p(x, \xi ; t-\tau) F(\xi, \tau) d \xi d \tau,
$$


where $p(x, \xi ; t-\tau)$ is the positive fundamental solution of $\mathcal{H}$ with singularity at $(\xi, \tau)$. By Proposition 3.3 we know that $w$ solves the problem

$$
\mathcal{H} w=-F \quad \text { in } \quad \mathbb{R}^{n} \times\left(0, t_{o}\right), \quad w(x, 0)=0, \quad x \in \mathbb{R}^{n} .
$$

Comparing (3.24), (3.25) and (3.27), we see that $v$ and $w$ solve the same Cauchy problem in the strip $\mathbb{R}^{n} \times\left(0, t_{o}\right)$. Furthermore, by the support properties of $\zeta$ we see that $v \in L^{\infty}\left(\mathbb{R}^{n} \times\left(0, t_{o}\right)\right)$. On the other hand, by the same reasons we have $F \in L^{\infty}\left(\mathbb{R}^{n} \times\left(0, t_{o}\right)\right)$, and the definition of $w$ gives for $\left.z=(x, t) \in \mathbb{R}^{n} \times\left(0, t_{o}\right)\right)$

$$
|w(z)| \leq\|F\|_{L^{\infty}\left(\mathbb{R}^{n} \times\left(0, t_{o}\right)\right)} \int_{0}^{t} \int_{\mathbb{R}^{n}} p(x, \xi ; t-\tau) d \xi d \tau \leq t_{o}\|F\|_{L^{\infty}\left(\mathbb{R}^{n} \times\left(0, t_{o}\right)\right)},
$$

since

$$
\int_{\mathbb{R}^{n}} p(x, \xi ; t-\tau) d \xi=1
$$

Thanks to Theorem 3.4 we can thus conclude that $v \equiv w$ in $\mathbb{R}^{n} \times\left(0, t_{o}\right)$, therefore

$$
v(z)=\int_{0}^{t} \int_{\mathbb{R}^{n}} p(x, \xi ; t-\tau) F(\xi, \tau) d \xi d \tau .
$$

Assume now that $z \in Q_{X}\left(z_{o}, r / 2\right)$. Since $\zeta(z)=1$, by the support properties of $p(x, \xi ; t-\tau)$ and $\zeta$ we obtain

$$
\begin{aligned}
u(z) & =\int_{t_{o}-4 r^{2}}^{t_{o}} \int_{B_{X}\left(x_{o}, 2 r\right)} p(x, \xi ; t-\tau) F(\xi, \tau) d \xi d \tau \\
& =\int_{t_{o}-4 r^{2}}^{t_{o}} \int_{B_{X}\left(x_{o}, 2 r\right)} p(x, \xi ; t-\tau) \mathcal{H} \zeta(\xi, \tau) u(\xi, \tau) d \xi d \tau \\
& +2 \int_{t_{o}-4 r^{2}}^{t_{o}} \int_{B_{X}\left(x_{o}, 2 r\right)} p(x, \xi ; t-\tau)<X \zeta(\xi, \tau), X u(\xi, \tau)>d \xi d \tau
\end{aligned}
$$

An integration by parts gives

$$
\begin{aligned}
& 2 \int_{t_{o}-4 r^{2}}^{t_{o}} \int_{B_{X}\left(x_{o}, 2 r\right)} p(x, \xi ; t-\tau)<X \zeta(\xi, \tau), X u(\xi, \tau)>d \xi d \tau \\
& =2 \sum_{j=1}^{m} \int_{t_{o}-4 r^{2}}^{t_{o}} \int_{\partial B_{X}\left(x_{o}, 2 r\right)} p(x, \xi ; t-\tau) X_{j} \zeta(\xi, \tau)<X_{j}, \nu_{\xi}>u(\xi, \tau) d \xi d \tau \\
& -2 \int_{t_{o}-4 r^{2}}^{t_{o}} \int_{B_{X}\left(x_{o}, 2 r\right)}<X p(x, \xi ; t-\tau), X \zeta(\xi, \tau)>u(\xi, \tau) d \xi d \tau \\
& -2 \sum_{j=1}^{m} \int_{t_{o}-4 r^{2}}^{t_{o}} \int_{B_{X}\left(x_{o}, 2 r\right)} p(x, \xi ; t-\tau) X_{j} X_{j} \zeta(\xi, \tau) u(\xi, \tau) d \xi d \tau
\end{aligned}
$$

where in the first integral in the right-hand side of (3.29) we have denoted by $\nu_{\xi}$ the spacial component of the outer unit normal to the smooth manifold $\partial B_{X}\left(x_{o}, 2 r\right)$. 
Since $X \zeta \equiv 0$ on $\partial B_{X}\left(x_{o}, 2 r\right) \times\left(t_{o}-4 r^{2}, t_{o}\right)$, the above integral vanishes, and after substituting (3.29) into (3.28) we obtain for $z \in Q_{X}\left(z_{o}, r / 2\right)$

$$
u(z)=\int_{Q_{X}\left(z_{o}, 2 r\right)} K(x, \xi ; t-\tau) u(\xi, \tau) d \xi d \tau,
$$

where

$$
\begin{aligned}
K(x, \xi ; t-\tau) & =p(x, \xi ; t-\tau) \mathcal{H} \zeta(\xi, \tau)-2<X p(x, \xi ; t-\tau), X \zeta(\xi, \tau)> \\
& -2 p(x, \xi ; t-\tau) \sum_{j=1}^{m} X_{j} X_{j} \zeta(\xi, \tau) \\
& =p(x, \xi ; t-\tau)\left\{\frac{\partial \zeta}{\partial \tau}+\sum_{j=1}^{m} X_{j} X_{j} \zeta-<\overrightarrow{\operatorname{div} X}, X \zeta>\right\}(\xi, \tau) \\
& -2<X p(x, \xi ; t-\tau), X \zeta(\xi, \tau)>,
\end{aligned}
$$

where we have denoted $\overrightarrow{\operatorname{div} X}=\left(\operatorname{div} X_{1}, \ldots, \operatorname{div} X_{m}\right)$. Recalling that $\zeta \equiv 1$ on $Q_{X}\left(z_{o}, r\right)$, and that $p(x, \xi ; t-\tau) \equiv 0$ when $\tau \geq t$, we see that for every fixed $z=(x, t) \in$ $Q_{X}\left(z_{o}, r / 2\right)$, the integral in (3.30) is actually performed on the region

$$
\left[Q_{X}\left(z_{o}, 2 r\right) \backslash Q_{X}\left(z_{o}, r\right)\right] \cap\left\{(\xi, \tau) \in \mathbb{R}^{n+1} \mid \tau<t\right\} .
$$

This observation will be important in the sequel. First of all, we can differentiate the right-hand side of (3.30) under the integral sign to obtain

$$
\begin{aligned}
& \sup _{Q_{X}\left(z_{o}, r / 2\right)}\left|\frac{\partial^{k}}{\partial t^{k}} X_{j_{1}} X_{j_{2}} \ldots X_{j_{s}} u\right| \\
& \leq \int_{Q_{X}\left(z_{o}, 2 r\right)}\left|\frac{\partial^{k}}{\partial t^{k}} X_{j_{1}} X_{j_{2}} \ldots X_{j_{s}} K(x, \xi ; t-\tau)\right||u(\xi, \tau)| d \xi d \tau .
\end{aligned}
$$

In what follows, to simplify the notation we will indicate with $X^{s} f$ the derivative $X_{j_{1}} X_{j_{2}} \ldots X_{j_{s}} f$ of a function $f$. Also, we write $\partial_{t} f$, instead of $\partial f / \partial t$. Leibniz rule gives

$$
\partial_{t}^{k} X^{s}(f g)=\sum_{i=0}^{k} \sum_{l=0}^{s}\left(\begin{array}{l}
k \\
i
\end{array}\right)\left(\begin{array}{l}
s \\
l
\end{array}\right)\left(\partial_{t}^{k-i} X^{s-l} f\right)\left(\partial_{t}^{i} X^{l} g\right) .
$$

Applying this formula to (3.31) we find

$$
\begin{aligned}
& \partial_{t}^{k} X^{s} K \\
& =\sum_{i=0}^{k} \sum_{l=0}^{s}\left(\begin{array}{l}
k \\
i
\end{array}\right)\left(\begin{array}{l}
s \\
l
\end{array}\right)\left(\partial_{t}^{k-i} X^{s-l} p\right)\left(\partial_{t}^{i} X^{l}\left\{\frac{\partial \zeta}{\partial \tau}+\sum_{j=1}^{m} X_{j} X_{j} \zeta-<\overrightarrow{\operatorname{div} X}, X \zeta>\right\}\right) \\
& +2 \sum_{j=1}^{m} \sum_{i=0}^{k} \sum_{l=0}^{s}\left(\begin{array}{l}
k \\
i
\end{array}\right)\left(\begin{array}{l}
s \\
l
\end{array}\right)\left(\partial_{t}^{k-i} X^{s-l} X_{j} p\right)\left(\partial_{t}^{i} X^{l} X_{j} \zeta\right) \\
& =I(x, t ; \xi, \tau)+I I(x, t ; \xi, \tau) \text {. }
\end{aligned}
$$


Substituting (3.34) in (3.33) we recognize that, in order to complete the proof of the theorem, it will suffice to establish to estimate

$$
\begin{aligned}
& \int_{Q_{X}\left(z_{o}, 2 r\right)}|I(x, t ; \xi, \tau)||u(\xi, \tau)| d \xi d \tau+\int_{Q_{X}\left(z_{o}, 2 r\right)}|I I(x, t ; \xi, \tau)||u(\xi, \tau)| d \xi d \tau \\
& \leq \frac{C}{r^{2 k+s}} \frac{1}{\left|Q_{X}\left(z_{o}, 2 r\right)\right|} \int_{Q_{X}\left(z_{o}, 2 r\right)}|u(\xi, \tau)| d \xi d \tau
\end{aligned}
$$

for some constant $C=C(X, s, k)>0$. We will prove that, in fact, each of the two terms in (3.35) is bounded by the quantity in the right-hand side. Also, since these two terms are similar we will only estimate one of them. Keeping in mind (3.32) we obtain from Lemma 3.2

$$
\begin{aligned}
& \int_{Q_{X}\left(z_{o}, 2 r\right)}|I(x, t ; \xi, \tau)||u(\xi, \tau)| d \xi d \tau \\
& \leq \sum_{i=0}^{k} \sum_{l=0}^{s}\left(\begin{array}{c}
k \\
i
\end{array}\right)\left(\begin{array}{l}
s \\
l
\end{array}\right) \frac{C(k, i, s, l)}{r^{2 i+l+2}} \\
& \times \int_{\left[Q_{X}\left(z_{o}, 2 r\right) \backslash Q_{X}\left(z_{o}, r\right)\right] \cap\{\tau<t\}}\left|\partial_{t}^{k-i} X^{s-l} p(x, \xi ; t-\tau)\right||u(\xi, \tau)| d \xi d \tau .
\end{aligned}
$$

We now break the integral in the right-hand side of (3.36) in two pieces

$$
\begin{aligned}
& \int_{\left[Q_{X}\left(z_{o}, 2 r\right) \backslash Q_{X}\left(z_{o}, r\right)\right] \cap\{\tau<t\}}\left|\partial_{t}^{k-i} X^{s-l} p(x, \xi ; t-\tau)\right||u(\xi, \tau)| d \xi d \tau \\
& \leq \int_{\left[Q_{X}\left(z_{o}, 2 r\right) \backslash Q_{X}\left(z_{o}, r\right)\right] \cap\left\{t_{o}-r^{2}<\tau<t\right\}}\left|\partial_{t}^{k-i} X^{s-l} p(x, \xi ; t-\tau)\right||u(\xi, \tau)| d \xi d \tau \\
& \int_{\left[Q_{X}\left(z_{o}, 2 r\right) \backslash Q_{X}\left(z_{o}, r\right)\right] \cap\left\{t_{o}-4 r^{2}<\tau<t_{o}-r^{2}\right\}}\left|\partial_{t}^{k-i} X^{s-l} p(x, \xi ; t-\tau)\right||u(\xi, \tau)| d \xi d \tau \\
& =I^{\prime}+I^{\prime \prime}
\end{aligned}
$$

To estimate $I^{\prime}$ we observe that since $t<t_{o}$ we can majorize

$$
\begin{aligned}
& I^{\prime} \leq \int_{\left[Q_{X}\left(z_{o}, 2 r\right) \backslash Q_{X}\left(z_{o}, r\right)\right] \cap\left\{t-r^{2}<\tau<t\right\}}\left|\partial_{t}^{k-i} X^{s-l} p(x, \xi ; t-\tau)\right||u(\xi, \tau)| d \xi d \tau \\
& \leq \int_{t-r^{2}}^{t} \int_{a r<d(x, \xi)<4 a r}\left|\partial_{t}^{k-i} X^{s-l} p(x, \xi ; t-\tau)\right||u(\xi, \tau)| d \xi d \tau
\end{aligned}
$$

where in the second integral we have used (2.9).

Theorem 2.6 implies

$$
\left|\partial_{t}^{k-i} X^{s-l} p(x, \xi ; t-\tau)\right| \leq \frac{C(k, i, s, l)}{(t-\tau)^{k-i+\frac{s-l}{2}}} \frac{1}{|B(x, \sqrt{t-\tau})|} \exp \left(-\frac{M d(x, \xi)^{2}}{t-\tau}\right) .
$$

We now observe that when $\tau>t-r^{2}$ we have from (2.6)

$$
|B(x, \sqrt{t-\tau})| \geq C_{1}\left(\frac{\sqrt{t-\tau}}{r}\right)^{Q}|B(x, r)| .
$$


Inserting (3.40) into (3.39) we find that when $\tau>t-r^{2}$ and $a r<d(x, \xi)<4 a r$

$$
\left|\partial_{t}^{k-i} X^{s-l} p(x, \xi ; t-\tau)\right| \leq \frac{C\left(k, i, s, l, C_{1}\right)}{(t-\tau)^{k-i+\frac{s-l+Q}{2}}} \frac{r^{Q}}{|B(x, r)|} \exp \left(-\frac{M a^{2} r^{2}}{t-\tau}\right)
$$

Substitution of (3.41) in (3.38) leads to the estimate

$$
\begin{aligned}
I^{\prime} & \leq C\left(k, i, s, l, C_{1}\right) \frac{r^{Q}}{|B(x, r)|} \sup _{t-r^{2}<\tau<t}\left\{\frac{1}{(t-\tau)^{k-i+\frac{s-l+Q}{2}}} \exp \left(-\frac{M a^{2} r^{2}}{t-\tau}\right)\right\} \\
& \times \int_{Q_{X}\left(z_{o}, 2 r\right)}|u(\xi, \tau)| d \xi d \tau . \\
& \leq \frac{r^{Q}}{|B(x, r)|} \frac{C^{\prime}(X, k, i, s, l)}{r^{2(k-i)+s-l+Q}} \int_{Q_{X}\left(z_{o}, 2 r\right)}|u(\xi, \tau)| d \xi d \tau . \\
& =\frac{C^{\prime}(X, k, i, s, l)}{r^{2(k-i)+s-l}} \frac{1}{|B(x, r)|} \int_{Q_{X}\left(z_{o}, 2 r\right)}|u(\xi, \tau)| d \xi d \tau .
\end{aligned}
$$

To estimate $I^{\prime \prime}$ we observe that, since $(x, t) \in Q_{X}\left(z_{o}, r / 2\right)$, on the region of integration we have $4 r^{2}>t-\tau>3 / 4 r^{2}$. Therefore, on such region the following estimate is a direct, and trivial, consequence of (3.39)

$$
\left|\partial_{t}^{k-i} X^{s-l} p(x, \xi ; t-\tau)\right| \leq \frac{C^{\prime \prime}(k, i, s, l)}{r^{2(k-i)+s-l}} \frac{1}{\left|B\left(x, \frac{\sqrt{3}}{2} r\right)\right|} .
$$

By (2.5) we conclude

$$
I^{\prime \prime} \leq \frac{C^{\prime}(X, k, i, s, l)}{r^{2(k-i)+s-l}} \frac{1}{|B(x, r)|} \int_{Q_{X}\left(z_{o}, 2 r\right)}|u(\xi, \tau)| d \xi d \tau .
$$

Combining (3.42), (3.44) with (3.37), and inserting the resulting inequality into (3.36) we conclude

$$
\begin{aligned}
& \int_{Q_{X}\left(z_{o}, 2 r\right)}|I(x, t ; \xi, \tau)||u(\xi, \tau)| d \xi d \tau \\
& \leq \frac{C(X, s, k)}{r^{2 k+s}} \frac{1}{r^{2}\left|B_{X}\left(x_{o}, 2 r\right)\right|} \int_{Q_{X}\left(z_{o}, 2 r\right)}|u(\xi, \tau)| d \xi d \tau .
\end{aligned}
$$

By analogous arguments, one obtains the following estimate

$$
\begin{aligned}
& \int_{Q_{X}\left(z_{o}, 2 r\right)}|I I(x, t ; \xi, \tau)||u(\xi, \tau)| d \xi d \tau \\
& \leq \frac{C(X, s, k)}{r^{2 k+s}} \frac{1}{r^{2}\left|B_{X}\left(x_{o}, 2 r\right)\right|} \int_{Q_{X}\left(z_{o}, 2 r\right)}|u(\xi, \tau)| d \xi d \tau .
\end{aligned}
$$

This completes the proof of the theorem.

\section{REFERENCES}

[Be] A. Bellä̈che, The tangent space in sub-Riemannian geometry. Sub-Riemannian geometry, Progr. Math., 144 (1996), Birkhäuser, pp. 1-78. 
[BLU] A. Bonfiglioli, E. Lanconelli \& F. UGuzzoni, Stratified Lie groups and potential theory for their sub-Laplacians, Springer Monographs in Mathematics. Springer, Berlin, 2007. xxvi+800.

[B] J. M. Bony, Principe du maximum, inégalité de Harnack et unicité du problème de Cauchy pour les operateurs elliptique degeneres, Ann. Inst. Fourier, Grenoble, 1, 119 (1969), pp. 277-304.

[CDG1] L. Capogna, D. Danielli \& N. Garofalo, Subelliptic mollifiers and a characterization of Rellich and Poincaré domains, Rend. Sem. Mat. Univ. Pol. Torino, 4, 54 (1993), pp. 361-386.

[CDG2] L. Capogna, D. Danielli \& N. Garofalo, The geometric Sobolev embedding for vector fields and the isoperimetric inequality, Comm. Anal. and Geom., 2 (1994), pp. 201215.

[CDG3] L. Capogna, D. Danielli \& N. Garofalo, Subelliptic mollifiers and a basic pointwise estimate of Poincaré type, Math. Zeit., 226 (1997), pp. 147-154.

[CG] L. Capogna \& N. Garofalo, Boundary behavior of nonegative solutions of subelliptic equations in NTA domains for Carnot-Carathéodory metrics, Journal of Fourier Anal. and Appl., 4, 4 (1995).

[CGN1] L. Capogna, N. Garofalo \& D. M. Nhieu, A version of a theorem of Dahlberg for the subelliptic Dirichlet problem, Math. Res. Letters, 5 (1998), pp. 541-549.

[CGN2] L. Capogna, N. Garofalo \& D. M. Nhieu, Properties of harmonic measures in the Dirichlet problem for nilpotent Lie groups of Heisenberg type, Amer. J. Math., 124 (2002), pp. 273-306.

[CGN3] L. Capogna, N. Garofalo \& D. M. Nhieu, Mutual absolute continuity of harmonic and surface measures for Hörmander type operators, Proc. Symposia Pure Math., Amer. Math. Soc., volume in honor of V. Maz'ya's 70th birthday, D. Mitrea, Ed., to appear.

[Ch] W.L. CHow, Über System von linearen partiellen Differentialgleichungen erster Ordnug, Math. Ann., 117 (1939), pp. 98-105.

[CGL] G. Citti, N. Garofalo \& E. LAnconelli, Harnack's inequality for sum of squares of vector fields plus a potential, Amer. J. Math., 3, 115 (1993), pp. 699-734.

[E] L. C. Evans, Partial Differential Equations, Grad. Studies in Math., Amer. Mat. Soc. vol.19, 1998.

[FSC] C. FEFFERMAn \& A. SAnChEz-CALle, Fundamental solutions for second order subelliptic operators, Ann. Math., 124 (1986), pp. 247-272.

[F] G. B. Folland, Subelliptic estimates and function spaces on nilpotent Lie groups, Ark. Math., 13 (1975), pp. 161-207.

[Fr] A. Friedman, Partial Differential Equations of Parabolic Type, Prentice-Hall, Inc., 1964.

[G] N. Garofalo, Analysis and Geometry of Carnot-Carathéodory Spaces, With Applications to Pde's, Birkhäuser, book in preparation.

[GN1] N. Garofalo \& D.M. Nhieu, Isoperimetric and Sobolev inequalities for CarnotCarathéodory spaces and the existence of minimal surfaces, Comm. Pure Appl. Math., 49 (1996), pp. 1081-1144.

[GN2] N. Garofalo \& D. M. NhiEU, Lipschitz continuity, global smooth approximations and extension theorems for Sobolev functions in Carnot-Carathéodory spaces, J. d'Analyse Math., 74 (1998), pp. 67-97.

[GS] N. GAROFALO \& F. SEgAla, Estimates of the fundamental solution and Wiener's criterion for the heat equation on the Heisenberg group, Indiana Univ. Math. J., 39 (1990), pp. $1155-1196$.

[Ga] B. Gaveau, Principe de moindre action, propagation de la chaleur et estimées sous elliptiques sur certains groupes nilpotents, Acta Math., 139 (1977), pp. 95-153.

[H] H. HöRmander, Hypoelliptic second-order differential equations, Acta Math., 119 (1967), pp. $147-171$.

[JSC] D. JERISOn \& A. SÁnchez-Calle, Estimates for the heat kernel for a sum of squares of vector fields, Indiana Univ. Math. J., 35 (1986), pp. 835-854.

[KS1] S. Kususoka \& D. W. Stroock, Applications of the Malliavin calculus, III, J. Fac. Sci. Univ. Tokyo, I A, Math., 38 (1987), pp. 391-442.

[KS2] S. Kususoka \& D. W. Stroock, Long time estimates for the heat kernel associated with a uniformly subelliptic symmetric second order operator, Annals of Math., 127 (1989), pp. $165-189$.

[LU1] E. Lanconelli \& F. Uguzzoni, On the Poisson kernel for the Kohn Laplacian, Rend. Mat. Appl. (7), 17 (1997), pp. 659-677.

[LU2] E. Lanconelli \& F. UGuzzoni, Degree theory for VMO maps on metric spaces and applications to Hörmander operators, Ann. Sc. Norm. Super. Pisa Cl. Sci. (5), 1 (2002), 
pp. 569-601.

[NSW] A. Nagel, E.M. Stein \& S. Wainger, Balls and metrics defined by vector fields I: basic properties, Acta Math., 155 (1985), pp. 103-147.

[Ra] P. K. Rashevsky, Any two points of a totally nonholonomic space may be connected by an admissible line, Uch. Zap. Ped. Inst. im. Liebknechta, Ser. Phys. Math. (Russian), 2 (1938), pp. 83-94.

[RS] L. P. RothsChild \& E. M. Stein, Hypoelliptic differential operators and nilpotent groups, Acta Math., 137 (1976), pp. 247-320.

[SC] A. SANCHEZ-CALLE, Fundamental solutions and geometry of sum of squares of vector fields, Inv. Math., 78 (1984), pp. 143-160.

[S] E.M. SteIn, Harmonic Analysis: Real Variable Methods, Orthogonality and Oscillatory Integrals, Princeton Univ. Press., (1993).

[VSC] N. Th. Varopoulos, L. Saloff-Coste \& T. Coulhon, Analysis and Geometry on Groups, Cambridge U. Press, 1992. 